\title{
EL DEPORTE EN LA PERSPECTIVA DEL AÑO 2000
}

\author{
Núria Puig \\ INEF-Catalunya. Passeig de l'Estadi s/n. 08004 Barcelona \\ Klaus Heinemann \\ Institut für Soziologie. Universität Hamburg. Allende Platz, 1, D 2000 Hamburg
}

Resum

L'article tracta sobre l'esport a l'any 2000. En primer lloc, es descriuen les tendencies que han caracteritzat el desenvolupament de l'esport durant els darrers desennis, tant les referides als tipus de pràctiques com al seu marc organitzatiu. A continuació, es valoren els canvis estructurals (demogràfics, configuració de la poblacib, estils de vida...) que generen aquesta situació. $A$ la conclusió s'examinen els impactes de les esmentades tendències a mig $i$ a llarg termini. A més, es posen de manifest els problemes que planteja fer una prospectiva, ja que es desconeixen molts dels paràmetres que caracteritzaran l'any 2000 (sistema politic, maneres d'emprar el temps liture, competència de les activitats esportives amb altres ofertes culturals...).

Resumen

El articulo trata sobre el deporte en el año 2000. En primer lugar, se describen las tendencias que han caracterizado el desarrollo del deporte en los ültimos decenios, tanto las referidas a modalidades de práctica como al marco organizativo. A continuacion, se valoran los cambios estructurales (demográficos, configuración de la población, estilos de vida...) que ban condicionado la emergencia de esta situación. En la conclusión se valoran los impactos de tales tendencias a medio y largo plazo. Se ponen de manifiesto, además, los problemas que surgen al intentar una prospectiva debido al desconocimiento que se tiene de algunos parámetros del año 2000 (sistema polttico, modos de utilizar el tiempo libre, competencia de las actividades deportivas con otras ofertas culturales....).

Abstract

The article treats with the sport in the year 2000 . In a first part it describes tendencies, which characterizes the development of the sport within the last decades; it describes the forms of sport practice as well as the structures of the organizations of sport. After that it discusses the structural (demografical, structure of the population, life-stile.., changes, which have obviously 
"Papers": Revista de Sociologia

influenced these developments. In a last part will be discussed the short and long run consequences of the described tendencies. But the article also has in mind the problems of a forecast due to the uncertainty we must have concerning the development of some parameters to the year 2000 (political system, development of the use of leisure time, competition of the sport with other cultural offers...).

\section{PLANTEAR UNA PERSPECTIVA DEL DEPORTE EN EL AÑO 2000: A MODO DE INTRODUCCION}

El presente artículo reffexiona sobre el futuro del deporte en el año 2000. Más allá de acontecimientos puntuales de mayor o menor espectacularidad - un ejemplo sería el caso de los Juegos Olímpicos de Barcelona- se pretende analizarlo en su globalidad incluyendo, por tanto, el conjunro de manifestaciones a través de las cuales se expresa.

Al no disponer de una teoría explicativa general sobre el desarrollo del deporte en la sociedad moderna - que pudiera proyectarse, en consecuencia, a los tiempos venideros - ha sido necesario emplear otro sisterna a la hora de plantear la prospectiva. En primer lugar, se han descrito las tendencias que han caracterizado el desarrollo del deporte en los últimos decenios; se han considerado los aspectos referidos a las modalidades de práctica y a las estructuras organizativas. Estas tendencias no son arbitrarias o casuales sino que han tenido lugar como consecuencia de unos cambios de orden estructural (demográficos, estructura de la población, configuración de los estilos de vida...) acaecidos en la sociedad. Ésta es la razón por la que se han examinado dichos cambios y su conexión con el deporte. Finalmente, se ha utilizado la escasa información disponible para analizar los impacros producidos por el pleno desarrollo de estas tendencias; era preciso este último paso en aras a plantear un escenario de futuro que fuera completo. El artículo, en consecuencia, ha sido estructurado siguiendo estas etapas.

Precisar también que se ha partido de una definición de deporte entendido como usistema abierto" (Dietrich\&Heinemann, 1989; Heinemann, 1986; 1991). La razón es que el deporte contemporáneo se muestra como una realidad cada vez más difícil de acotar, más imprecisa. Constantemente aparecen nuevas prácticas que, aún no ajustándose a concepciones clásicas del deporte, tienen que ver con la actividad física y, por tanto, es necesario tomarlas en consideración.

En este sentido, es oportuno recordar que, en uno de sus últimos escritos - publicado precisamente en esta misma revista-, José María Cagigal ya planteaba la necesidad de cuestionarse anteriores defniciones del deporte por 
considerarlas reduccionistas (Cagigal, 1983). Recomendaba orientar la reflexión entendiendo el deporte como un fenómeno cultural y, por tanto, permeable a las transformaciones del entorno. Esta ha sido la opción realizada. Considerar el deporte desde una perspectiva polisémica (Brohm, 1976), como un sistema complejo con diversidades y contradicciones. Se han tenido en cuenta, por tanto, el conjunto de actividades físicas que las personas de las sociedades modernas realizan durante su tiempo de ocio. Ello no impide que, a la hora de efectuar investigaciones empíricas puntuales, se puedan fijar criterios operativos y se hagan definiciones coyunturales adecuadas a cada circunstancia.

\section{DIVERSIFICACIONES Y OPOSICIONES}

\section{El deporte tradicional}

La tendencia más relevante del sistema deportivo contemporáneo es la de su diversificación. El deporte ha dejado de ser un sistema autónomo y se ha convertido en un sistema abierto con escasa identidad propia y estrechamente conectado a otros sistemas tales como el económico, el educativo, el político... (gráfico 1).

\section{GRÁFICO I}

La transformación en el deporte

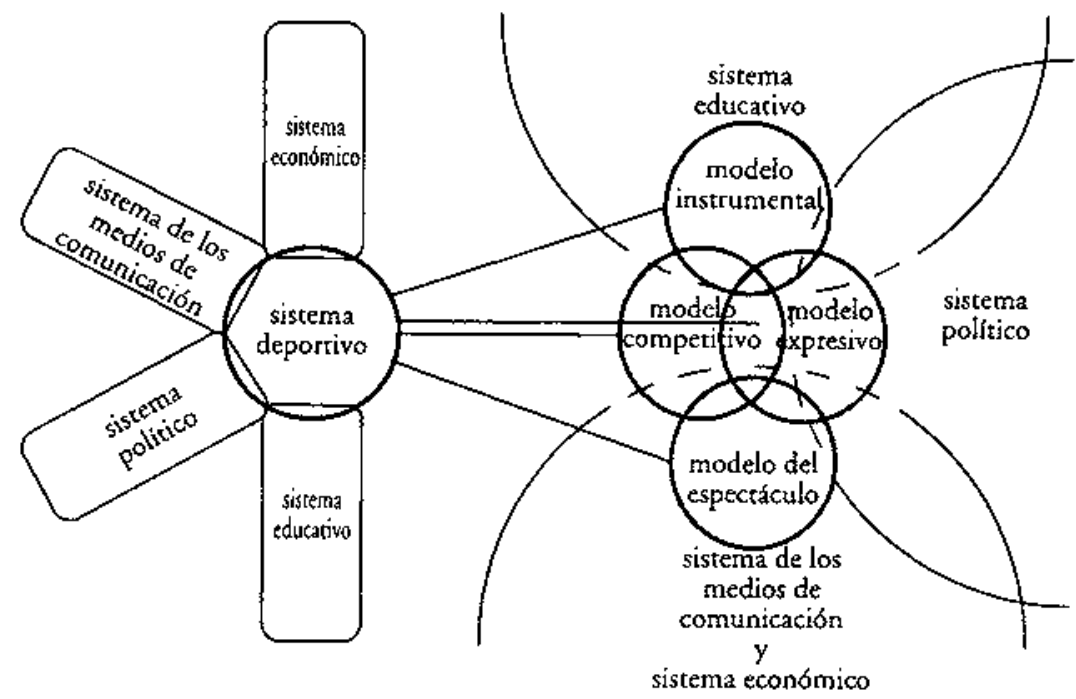


El deporte tradicional era una unidad homogénea y una entidad autónoma que ya no explica la realidad actual del fenómeno (Heinemann, 1991). Se asociaba a metas claramente definidas en el ámbito de reglamentaciones perfectamente claras. La idea era siempre conseguir ciertos logros en base a una preparación disciplinada y contínua. Deporte significaba competitividad y orientación hacia una meta o logro.

Tenia tambien una uniformidad de estructuras de valor. Combinaba valores individuales como la camaradería, el espíritu comunitario, la solidaridad... con el logro, la diversión, el equipo, la competición... La experiencia del juego y el juicio de los resultados eran, en consecuencia, siempre inseparables.

El marco a través del cual podía llevarse a cabo este tipo de deporte era el club deportivo, con elementos de trabajo voluntario, estructura democrática decisoria, independencia de terceras partes, símbolos identificadores... Por último, tanto por el tipo de prácticas que ofrecía como por el marco organizativo que las acogía, este deporte generaba procesos de selección y marginación. Sólo se integraban al mismo aquellas personas cuyas posibilidades de acción e intereses coincidían con sus características fundamentales. Era un coto cerrado para jóvenes varones de las clases media y alta. Más arractivo para jóvenes que para adultos, para hombres que para mujeres...

La situación ha cambiado. El análisis de la población que practica deporte y las organizaciones deportivas ya no se ajusta a la descripción efectuada. Se ha producido una ruptura del modelo tradicional.

\section{Modelos diferenciados}

El deporte contemporáneo se caracteriza por un proceso de diferenciación creciente. Ya no hay un solo modelo explicativo que agrupe sus características principales. Si se tienen en cuenta como dimensiones constituyentes la forma como se organizan las actividades deportivas, el modo como se legitiman, las motivaciones de los participantes y los impactos que producen, cabe sugerir cuatro modelos conffguradores del deporte contemporáneo. Se trata de los siguientes: competitivo, expresivo, instrumentaly espectáculo. Dichos modelos deben ser entendidos como "tipos ideales", según el modo como fueron descritos por Max Weber. Se trata de abstracciones, de polarizaciones, a las cuales se refieren más o menos determinadas manifestaciones del deporte contemporáneo. Así, el modelo competitivo contiene elementos expresivos, el instrumental puede llegar a combinarse con el espectáculo... Lo importante es que, en su conjunto, den cuenta de una realidad.

El modelo competitivo del deporte es el heredero directo del deporte tradicional. Mantiene, por tanto, una reglamentación estricta y universal para 
todos los participantes, una uniformidad de las estructuras de valores (fairplay, espiritu de equipo, experiencia de ganar o perder...), y una estructura organizativa fundamentada en el club (trabajo voluntario, estructura democrática decisoria, simbología propia...). Se orienta en su conjunto a la consecución de una meta y se legitima precisamente por los logros alcanzados o que se pretenden alcanzar: una medalla, una victoria, la integración social de los marginados, la educación de la juventud... Concuerda con la ética del trabajo, con la idea que las personas se realizan por su proyección futura, por los logros alcanzados. Sigue siendo el coto para jóvenes deportistas pese a que, probablemente debido a la mayor penetración del deporte en la sociedad contemporánea, se ha extendido hacia otros sectores sociales y ha incorporado a mayor número de mujeres jóvenes identificadas con los valores del modelo.

El contraste con el modelo expresivo es notorio. Éste último, representa, en el fondo, un cambio de valores en las sociedades modernas. Este modelo engloba prácticas escasamente organizadas y sometidas a procesos constantes de innovación y diversificación. Así, ir a esquiar a los Alpes tiene un significado mucho más amplio que en tiempos anteriores. Quienes viajan a esta zona, si bien es cierto que esquiarán, también harán surf, mono-esquí, parapente con esquíes, ascenderán una montaña andando o en helicóptero... El modelo agrupa, por tanto, actividades muy variadas: navegar por el Báltico, hacer windsurf en Tarifa, disfrutar un trekking en el Himalaya, aprender a bailar sevillanas o asistir a cursos de yoga, tai-chi o meditacion trascendental... Se fundamenta en el goce por el presente (having fun, having pleasure...) y, en muchos casos, se plantea como una compensación frente a la excesiva planificación de la vida cotidiana. En consecuencia, no se legitima por los resultados a alcanzar, por las realizaciones, sino por la gratificación íntima que cada persona obtiene en su práctica.

El modelo instrumental se asocia generalmente con empresas comerciales que disponen de sofisticada maquinaria (las body-machines) que permite el cultivo del cuerpo en aras a darle una forma más acorde con determinadas concepciones. En este caso, se espera obtener gratificación por la respuesta que los demás darán ante el cuerpo que uno o una muestre. La preocupación casi obsesiva por la salud o el envejecimiento es un componente muy importante en las motivaciones de quienes practican. En orros casos, además, se va más allá y se asocia el cuterpo a la única forma como unos y otras se dan identidad. American psycho, la reciente novela de Bret Easton Ellis, es un exponente - llevado hasta las últimas consecuencias- de este modelo. Las personas no son más que apariencia; en consecuencia, la forma del cuerpo y los vestidos y accesorios que lo cubren son lo único que cuenta. Se respeta o se desprecia - hasta el extremo de asesinarla - toda persona que no 
responda a este tipo de requerimiento. El gimnasio, adernás, forma parte esencial del modo de vida del protagonista $y$, por supuesto, este lugar no puede tener otro nombre que "Exclusive».

El modelo del espectáculo no es nuevo (siempre han existido espectáculos deportivos) pero tiende cada vez más a regirse por las leyes del mercado. La ley del deporte, recientemente aprobada por el Parlamento español, así lo refleja al obligar a los clubes profesionales a convertirse en sociedades anónimas. Se trata, por tanto, de que funcionen como cualquier otra empresa Iucrativa sometida a un tipo de jurisdicción común a todas ellas. En su dimensión deportiva - que, por supuesto, se sigue manteniendo- el modelo del deporte-espectáculo cuenta con una reglamentación estricta (reglamentos de federaciones, control antidoping, medidas contra la violencia en los estadios...) y un cuerpo de profesionales que se ocupan de su cumplimiento (jueces, árbitros, comités de disciplina...). Es un modelo orientado al entertainement y sus formas de legitimación difieren mucho de las de los anteriores. Su fomento se justifica por un afán lucrativo (NBA u otras ligas profesionales), por impulsar el desarrollo de una ciudad o un país (el caso de los Juegos Olímpicos de Barcelona '92), por reconstruir identidades en áreas periféricas de grandes áreas metropolitanas (Callède, 1984; Puig; Burriel y col, 1990), por ensalzar las virtudes de un Estado (Cuba, los del antiguo bloque socialista...) o por la combinación de dos o más de estos argumentos. Por el contrario, el recurso a la salud dista mucho de estar en el orígen de la justificación de este modelo; las voces que claman por lo poco saludable que resulta el deporte asociado a la búsqueda de la performance -incluyendo en este contexto la problemática del dopping - son cada vez más frecuentes.

\section{Caracterización a partir de oposiciones}

Además de las características que los definen internamente, los modelos expuestos se diferencian entre sí por el posicionamiento que adoptan en relacion a cinco dimensiones que caracterizan el conjunto del sistema deportivo contemporáneo. Dimensiones cuya característica principal consiste en plantearse a partir de polaridades. Así, en contraste con formas tradicionales de deporte, el deporte contemporáneo es a la vez: unifuncionalidad de objetivos/multifuncionalidad de objetivos, competición/no-competición, performance/no-performance, reglamentación/no-reglamentación, internacional/local y organizado/no-organizado.

El interés de analizar los modelos desde esta perspectiva es que les da dinamismo y permite examinar el modo como cada una de las prácticas asociadas a uno u otro cristaliza concretamente en las dimensiones expuestas. Tambien permite conocer la mayor o menor proximidad de cada una de es- 
tas prácticas al modelo de referencia o ver como, pese a la misma denominación, una actividad remite a más de un modelo.

Tómese el ejemplo del windsurf en el mar. Sus inicios van muy unidos al modelo expresivo: el placer por deslizarse sobre las olas con la ayuda del viento, la pausa al mediodía en el curso de una intensa vida laboral o el complemento a una reposada estancia en una lejana playa del Caribe... Con el tiempo, la actividad ha sufrido un proceso de diferenciación y, en este sentido, algunas formas de windsurf han incorporado elementos competitivos, con el objetivo de ganar, de alcanzar la performance, con reglamento en unas competiciones internacionales convenientemente organizadas; el modelo tradicional, en definitiva. Se observa, en consecuencia, cómo las actividades deportivas sufren diversificaciones y deslizamientos entre las polaridades planteadas por las dimensiones expuestas (gráfico 2).

El sistema abierto en que se ha convertido el deporte contemporáneo ha tenido efectos en su estructura organizativa. En realidad, ésta no es más que una variable dependiente y, cambiando el contexto donde se ha generado, los cambios en su propia estructura son la consecuencia lógica. Lo que ahora no son más que indicios puede ser el anuncio de un cambio radical en la estructura deportiva del futuro.

\section{GRÁFICO 2}

Posicionamiento de actividades deportivas según las dimensiones del deporte contemporáneo

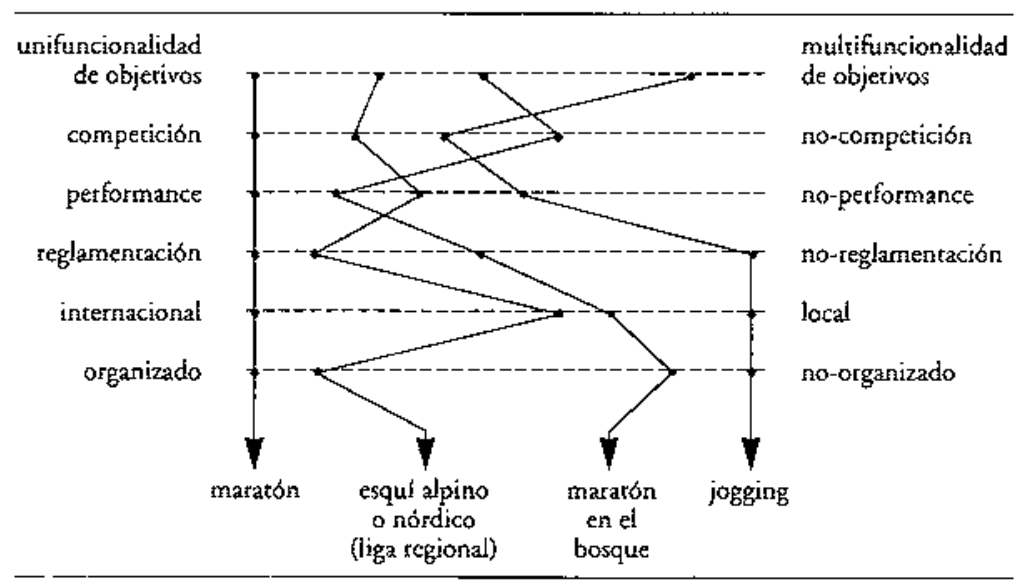


«Papers»: Revista de Sociologia

CAMBIOS EN LA ESTRUCTURA ORGANIZATIVA DEL DEPORTE

\section{Hacia un asociacionismo instrumental}

Se observan inestabilidades y cambios en el movimiento asociativo, en general, y el deportivo, en particular. Se sabe de clubs que desaparecen porque la disminución de personas asociadas ha hecho inviable su existencia; clubs de "toda la vida" muchos de los cuales habían sobrepasado los cincuenta años.

Este sentir general se contradice con otras informaciones procedentes de investigaciones efectuadas. En primer lugar, el asociacionismo deportivo $\mathrm{fl}_{1-}$ gura entre los más importantes. En el Ârea Metropolitana de Barcelona un $39,5 \%$ de las personas están asociadas. De ellas, un 36\% lo están a un club deportivo (Institut d'Estudis Metropolitans, 1986). En cuanto a la población juvenil de Barcelona, el $30 \%$ pertenece a una asociación; un $60 \%$ de este grupo está adherido a una de carácter deportivo (Jiménez y col, 1990). Se está produciendo, además, una gran mobilidad en el mundo asociativo. Si bien tan sólo el $30 \%$ de la población juvenil barcelonesa está asociada, el $61,6 \%$ de la misma afirma tener experiencia asociativa. Se observa un movimiento de entradas y salidas muy elevado, o corrientes de población hacia determinados tipos de asociaciones en detrimento de otros. Fenómenos similares se observan en todos los páses industrializados (Heinilä, 1989; Horch, 1989; Malenfant, 1987, 1989; Slack\&Kikulis, 1989).

En realidad, lo que está sucediendo es un cambio de valores en el mundo asociativo. La cultura asociativa, al igual que el modelo deportivo, ya no es homogénea sino que se está diversificando. Las organizaciones tradicionales -los clubs- tienen problemas; paralelamente, sin embargo, surgen nuevas formas organizativas del deporte. La encuesta sobre asociacionismo juvenil en Barcelona es de nuevo ilustrativa a este respecto. No se trató el asociacionismo deportivo como una sola categoría sino que se diferenció entre uservicios deportivos y de mantenimiento físicon y "deportes de equipo y práctica deportiva»; el número de personas adheridas era de un $41,3 \%$ y un $22,8 \%$, respectivamente. El dato es muy ilustrativo de lo que se comenta, puesto que la forma menos convencional de asociacionismo es la que predomina. Resultados similares se han obtenido en otro estudio - en este caso de carácter cualitativo- sobre jóvenes y deporte también realizado en la ciudad de Barcelona (Puig, 1992).

En resumen, se puede afirmar que gran parte de la población requiere más, de la organización donde acude, un servicio que un lugar de sociabilidad entendido en el sentido convencional; más marketing y profesionalidad en la oferta de servicios que voluntarismo. El asociacionismo deportivo - así como el asociacionismo en general - tiende a acentuar su valor instrumental 
El deporte en la perspectiva del año 2000

en contraposición a valores más centrados en sentimientos de pertenencia que hasta ahora predominaban.

\section{Diversificacion organizativa}

La cultura de una organización ha venido caracterizándose a partir de las tres dimensiones siguientes (Heinemann, 1992) (gráfico 3):

1. Comportamiento corporativo. Cada asociación genera unos modos de relación entre las personas que la componen basada en una estructura de valores (el amor a la montaña, lo que se puede hacer y lo que no en los locales de reunión, formas más o menos innovadoras de financiamiento...), una ideología (catalanista, españolista, liberal...), una forma de legitimación (el fomento del respeto por la naturaleza, la integración de las poblaciones marginales...), un modo de enseñar la práctica deportiva —o las prácticas- de su competencia ("escuela de campeones", "el deporte a la medida de cada cualm...).

2. Simbolos corporativos. Escudos, banderas, vestimenta, colores, arquitectura...

\section{GRÁFICO 3}

El desarrollo de las organizaciones deportivas

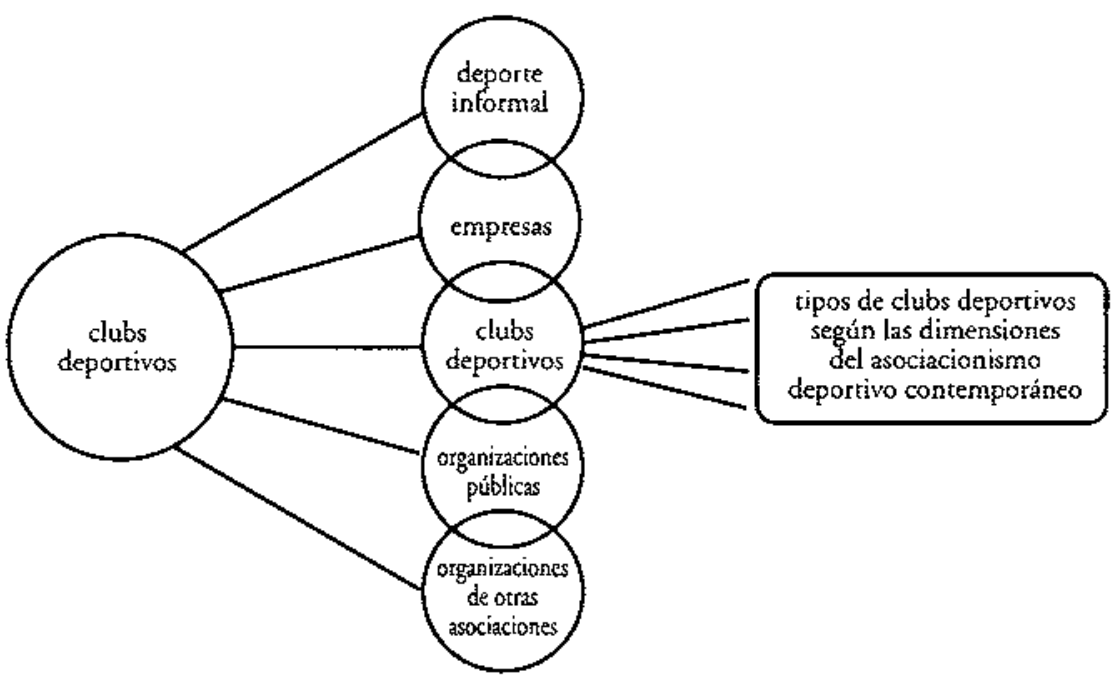


"Papers»: Revista de Sociologia

\section{GRÁFICO 4}

Esquema configurador del asociacionismo deportivo contemporáneo

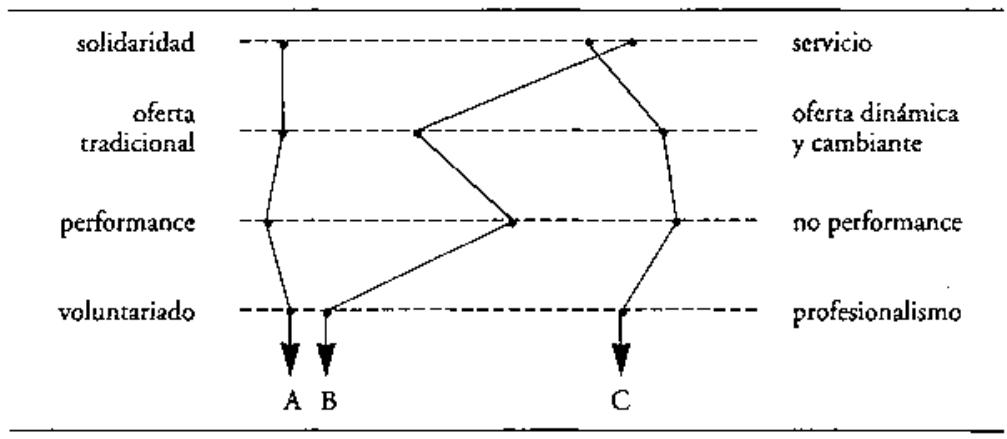

3. Comunicación corporativa o el modo como la asociación se presenta ante los demás. Se puede ofrecer una imagen de distinción, de austeridad, de innovación, de ambiente simpático y relajado, de fraternidad...

La combinación de estos aspectos da lugar a que, con el tiempo, cada asociación haya generado una cultura propia mediante la cual es distinguida y se distingue de las demás. Sin embargo, pese a esta imagen distintiva que cada club se otorga, puede considerarse que el asociacionismo deportivo tradicional ha gozado de unas caracrerísticas bastante homogéneas (solidaridad, voluntarismo, pràctica deportiva más o menos orientada a alcanzar un objerivo...). Actualmente, al igual como ha sucedido con los modelos de práctica, se asiste a una progresiva diferenciación en las culturas que caracterizan las organizaciones deportivas (gráfico 4). Tal fenómeno también se articula en torno a cuatro polaridades.

Por solidaridad se entiende una forma de integración al club basada en circunstancias emocionales; es una forma emocional de integración. Crea sentimientos de pertenencia que se sustentan en anécdotas del pasado, en el deber y otras formas de afecto hacia los demás, en la idea de pertenecer a un colectivo estructurado más allá de la historia individual... Esta forma de integración se opone a la que acontece cuando la asociación se orienta más a la oferta de servicios. Quien se integra a ella es porque se le ofrece un producto acorde con sus aspiraciones individuales. El sentimiento de pertenen- 
cia puede surgir con el paso del tiempo pero, ante todo, prima el valor instrumental de la asociación.

La oferta que efectúan las asociaciones también oscila entre el polo de la tradición y el cambio permanente. Los clubs tradicionales suelen hacer una oferta uniforme muy orientada a garantizar la práctica de los equipos que les representan o de los socios y socias que siempre han practicado. No buscan nueva clientela o, más bien, la cultura organizativa que han generado con el paso del tiempo, les impide ser ágiles para introducir cambios en su oferta. En el otro extremo se encuentran organizaciones especializadas en atender cualquier demanda llegando -o intentándolo hacer- a satisfacer toda nueva necesidad individual que surja.

La lógica de la performance puede contraponerse a la idea de jugar o divertirse más allá de ganar o perder. Los clubs deportivos tambien oscilan a lo largo de este eje, tendiendo a diversificar el tipo de objetivos que se pueden alcanzar a través de la práctica deportiva. En el mismo sentido, en aras a facilitar el acceso de un público más amplio, y también para organizar el trabajo del club de modo más sistemático y racional, se tiende a la profesioralización de algunos de sus técnicos.

En el momento actual, las asociaciones deportivas se hallan confrontadas a estas polaridades y, de modo consciente o no, se ven obligadas a posicionarse en relación a ellas. Es ya difícil que un club responda al modelo estrictamente tradicional; sin embargo, cada vez son más frecuentes los que tienen. situaciones intermedias en los ejes descritos. En general, se da la búsqueda de un espacio propio mediante el cual la cultura organizativa siga subsistiendo a pesar de los retos de la modernidad. Los clubs que no comprenden la necesidad de una cierta adaptación al signo de los tiempos tienen graves dificultades para subsistir.

Y el recurso a la ayuda pública puede paliar ciertas insuficiencias pero, a largo plazo, no rompe con la tendencia que se está dando. Pese a que la Constitución espańola establezca que los poderes públicos fomentarán la educación física y el deporte (artículo 43.3), los recursos no son ilimitados - el gasto público ha aumentado de modo alarmante en pocos años (Burriel, 1991) - y ello obliga a establecer prioridades; es obvio que las actividades físicas no lo son. Así, tanto si es mediante la concesión de subvenciones, como por las directrices establecidas en sus servicios de competencia, los poderes públicos tienden a introducir criterios de racionalidad económica en sus actuaciones deportivas (Burriel, 1991). Aunque no excluyan la idea de rentabilidad social, se ven obligados a "adaptarse a los signos de los tiempos", diversificar la oferta a todos los públicos posibles para hacerla rentable en la medida de lo posible. La gratuidad del deporte, que se llegó a imponer como criterio en los años de la transición, ha sido puesta en duda seriamente. 
"Papers»: Revista de Sociologia

En este contexto se desenvuelven actualmente las asociaciones deportivas. Se asiste a un cambio en las estructuras organizativas del deporte, generadas por cambios en el modelo deportivo tradicional. Cambios que remiten a las profundas transformaciones generales que han acaecido en los últimos decenios.

\section{TRANSFORMACIONES EN LA SOCIEDAD CONTEMPORÁNEA}

Las tendencias analizadas tienen su orígen en cambios más profundos de orden general. No habrían sido presentadas como tales y con proyección de futuro si no fuera por este motivo. La importancia de los cambios es tan profunda que confirma y da solidez a los pronósticos efectuados. Por este motivo, se analizan en detalle.

\section{Cambios en la estructura de la población}

Las tasas de natalidad en los países avanzados han disminuído ostensiblemente; en España este fenómeno ha sido especialmente relevante. En conjunto se está produciendo un envejecimiento de la población. Ello conduce, obviamente, a un cambio en el perfil de quienes practican deporte. Los modos de practicar se adaptan a las características de cada cual; es, en definitiva, una transformación del modelo tradicional.

Sin embargo, el impacto mayor en el cambio de hábitos deportivos, desde el punto de vista de la estructura de la población, también debe hallarse en el aumento de los niveles educativos y los cambios en el núcleo familiar. Es sabido que la práctica del deporre tiene mayor arraigo entre los sectores con mayor nivel de instrucción. La percepción de los valores positivos del deporte (ranto del punto de vista de la salud como del de la distinción social adquirida a través del mismo) es mayor en estos grupos. En consecuencia, un aumento generalizado de los niveles educativos de la población genera mayor participación deportiva.

Asimismo, la transformación del núcleo familiar ha repercutido en la emergencia de los modelos deportivos. La familia tradicional pierde importancia frente a otras formas de convivencia. Personas que viven solas; incremento de los divorcios o separaciones acompañado, entre otras cosas, del attmento de mujeres que asumen la responsabilidad familiar superando las incidencias de la vida cotidiana; población juvenil que, en mayor o menor medida, busca formas alternativas - o diferenciadoras- de vida en las cuales se incorpora el deporte. María Angeles Durán (1987) llama la atención sobre el caso de las mujeres separadas. Representan un grupo en franca ruptura con 
los hábitos tradicionales; se han visto obligadas a asumir roles llamados masculinos, aspecto que ha generado una profunda transformación de sus modos de vida. Los datos disponibles indican que sus indices de práctica deportiva son superiores a los de la media de las mujeres; parecería como si el deporte, y el marco en el cual se desenvuelve tal actividad, contribuyera a reforzar lazos de solidaridad entre mujeres $y$, en último término, a una revalorización de sí mismas.

\section{Reduccion de la jomada de trabajo}

El tiempo dedicado al trabajo ha ido reduciéndose considerablemente desde principios de siglo. En términos generales, hacia 1900 era corriente trabajar cerca de 60 horas semanales, mientras que, en la actualidad, la cifra de 35 se halla más o menos extendida en los países objeto del presente análisis. Más importante todavía es el periodo de actividad profesional que también se ha reducido, sobre todo si se tiene en cuenta el aumento de la esperanza de vida. Así, la inserción en el mundo laboral es más tardía, con lo cual los años de juventud, de transición, de inestabilidad... son más largos y conllevan un modo de vida distinto al de quienes forman parte del mundo del trabajo propiamente dicho.

La vida de los años profesionales adquiere otros significados, los cuales también se traducen en otros hábitos que, por supuesto, pueden incluir los deportivos. Y lo mismo ocurre con los años posteriores a la jubilación, que ya no es el "final" sino que, pese a los impactos psicológicos iniciales que se producen en muchas personas, se planrean como el tiempo para hacer todo aquello que no se pudo hacer antes (escribir, viajar, hacer más deporte, aprender...).

\section{Aumento del bienestar}

Es preciso destacar, también, el aumento del bienestar. No sólo se ha producido un aumento generalizado de los ingresos sino que, de modo muy extendido, se ha ido produciendo la constitución de patrimonios familiares (via herencia o gracias a la posibilidad de efectuar compras mediante créditos) que, con los años, han liberado una parte de los salarios, Ia cual ha podido ser inverrida en actividades de ocio en general. No hay síntomas de que esta tendencia vaya a desaparecer. En Alemania, por ejemplo, se calcula que para el año 2000 tan sólo un $30 \%$ del salario de una persona adulta estará destinado a gastos fijos. Por supuesto que tal consideración debe ser muy matizada según los países; para el caso español no se pueden dar datos tan esperanzadores. Sin embargo, no es menos cierto que, con mayor o menor 
endeudamiento, se ha producido un aumento del bienestar en los hogares españoles, que permite orientar ciertos recursos e intereses hacia actividades de ocio.

Tanto la reducción de la jornada de trabajo como el aumento del bienestar han producido cambios cuantitativos y cualitativos de interés. No sólo es importante que haya más tiempo disponibie (lo cual, por otro lado, no es tan evidente debido, entre otras cosas, al aumento del tiempo de transporte en las grandes áreas metropolitanas) sino que, por encima de todo, se asiste a una diversificación de los sectores de la población sometidos a situaciones coyunturales bien diferentes. $Y$ todo ello en un contexto general de mejora de la calidad de vida. En conjunto, se dan unas condiciones favorables a la emergencia de estilos de vida diferenciados, que integrarán y generarán, en mayor o menor medida, los modelos deportivos analizados en páginas anteriores.

\section{Diferenciación e individualización de los estilos de vida}

Pese a las dificultades metodológicas que plantea su estudio, existe el común acuerdo que las variables explicativas tradicionales (sexo, edad, niveles de instrucción...) son insuficientes para dar cuenta de las formas de vida en las sociedades industrializadas. Al tiempo que el principio del conocimiento teórico y de la racionalidad económica se impone, surge el que aboga por el disfrute de la propia individualidad -el de la realización personal-- a través de prácticas culturales (leer, viajar, hacer deporte, oír música...) (Bell, 1976; García Ferrando 1991a). Este último principio justifica la importancia de los factores culturales como elementos explicativos de las formas de vida contemporáneas. Su centralidad aumenta y, en este contexto, el tiempo libre - como momento óptimo para la autorrealización-cambia de significado; no es residual, es imprescindible para la dinámica social. Se valora y se acepta la autorrealización personal por encima de concepciones más rígidas e impuestas que predominaban en tiempos anteriores. El peso de las instituciones (la Iglesia, la escuela...) es mediatizado por la tolerancia general hacia comportamientos más individualizados, porque a través de ellos cada persona puede realizarse. Ello conlleva una diversificación de los estilos de vida y, en consecuencia, de las prácticas que los integran. El deporte entra de pleno en este proceso de diversificación (García Ferrando, 1991a; Heinemann, 1986).

En España, Andrés Orizo (1979) y García Ferrando y Pérez de Guzmán (1990), entre otros, han investigado sobre la cuestión aportando tipologías variadas de estilos de vida. Pese a los problemas metodológicos que los mismos autores plantean, es posible observar como no todos los estilos de vida integran por igual el deporte y como, en general, son los más innovadores 
quienes lo hacen preferentemente. Ello significa que el deporte, por el momento, figura como una práctica en alza, valorada positivamente, elemento de distinción y configurador de identidades; debe añadirse a ello una revalorización general del cuerpo y el interés crecienre por el cuidado de la salud. Para cumplir tal requisito es necesario que la forma deportiva se adapte a todos los esquemas de valores existentes, y no sólo a los tradicionales, más centrados en la moral del trabajo, la proyección de futuro en aras a un objetivo concreto... Otros valores, tales como vivir el presente, realizarse en el ocio y no en el trabajo... también están presentes. Los modelos deportivos ana. lizados son, en consecuencia, producto de esta diversificación de los estilos de vida.

\section{LOS IMPACTOS: A MODO DE CONCLUSIÓN}

Para comprender el deporte en el año 2000 será preciso tener en cuenta la diversificación del sistema deportivo tradicional y su evolución hacia sistema abierro. Cada uno de los modelos analizados estará sometido a la lógica de otros sistemas sociales (económico, político, educativo...) de tal modo que dejará de tener una lógica propia.

Ahora bien, el análisis efectuado sería insuficiente si no se tuvieran en cuenta los impactos de todo tipo que pueden surgir como consecuencia de las transformaciones acaecidas. Por nuevos, desconocidos, lo cual obliga a prestarles cada día mayor atención con el fin de preveer evoluciones futuras. La documentación disponible a este respecto es, por el momento, escasa. Se ha intentado sacar el máximo partido a la misma con el fin de perfilar el modo como podrían orientarse estudios sobre la cuestión.

El aumento de los índices de participación deportiva que se observan periodicamente se explican por la diversificación a que hemos venido aludiendo (García Ferrando, 1982, 1986, 1991b). En este sentido, en los últimos estudios realizados se aprecia un mayor incremento de la participación femenina en relación a la masculina (García Ferrando 1991b; Vázquez, 1990). La heterogeneidad de modelos favorece que cada cual pueda sentirse atraído por alguno, sin, por ello, romper con sus concepciones del cuerpo, la salud, el placer...

De entrada, un incremento en la participación deportiva y el interés por el deporte significa un impacto económico positivo. El deporte es una referencia cultural que se compra (espectáculos deportivos, vestimenta, servicios, material, clases...) y también sirve para vender (publicidad, sponsoring, patrocinio...) (Bale, 1989; Heinemann, 1992; Metoudi, 1987). El mercado de trabajo del deporte es un sector en expansión y, en España, se 
estima que tan sólo en los sectores de la docencia, entrenamiento, animación y dirección ocupa al $0,3 \%$ de la población activa (Consejo Superior de Deportes, 1991). Por el momento se desconocen cifras sobre empleos generados por el impacto del deporre en el mundo de la industria y los servicios. El porcentaje referido a la población activa empleada en este sector aumentaría bastante sin duda.

El tema adquiere complejidad cuando se examinan otras consecuencias. La marcada tendencia a la individualización está creando serias dificultades al modelo organizativo tradicional, uno de cuyos ejes constitutivos es la solidaridad. Hace 30 años, el 90\% de quienes practicaban deporte en Alemania pertenecían a una asociación deportiva; actualmente, la cifra ha disminuído hasta un $25 \%$. ¿Se conocen exactamente las consecuencias de esta udictadura de las decisiones individualesn?, ¿conducirá ello a la desaparición de formas de identidad colectiva y de los lazos de solidaridad entre comunidades? Preguntas similares pueden hacerse respecto a la profesionalización creciente de las asociaciones deportivas. Por un lado, parece necesaria en aras a una mejora de la gestión; sin embargo, por el otro, estudios realizados muestran como la desaparición del voluntariado también rompe con el dinamismo de dichas asociaciones (Koski, 1991). Queda por ver la evolución que seguirán los modelos analizados y las consecuencias que puedan derivarse de la predominancia de unos sobre los otros.

Además, el problema reside en el encadenamiento de impactos que se pueda producir. Ante determinadas decisiones, es muy dificil predecir las consecuencias que a medio o largo plazo puedan tener. Así, por ejemplo, el deporte, junto al turismo, se muestra como instrumento privilegiado para la reactivación económica de áreas depresivas. Tanto en la montaña como en las zonas marítimas permite hacer ofertas atractivas para poblaciones ávidas de avenrura, descanso, distracción, ejercicio...; los resultados están siendo buenos. Al mismo tiempo, sin embargo, se alerta sobre las consecuencias ecológicas de determinadas intervenciones. $\mathrm{El}$ trial en la montaña genera polución no exenta de contaminantes químicos, las barcas motoras desprenden aceite y gasolina en los mares, las pistas de esquí alpino crean etosiones en las zonas de montaña que afectan el equilibrio de los ecosistemas... (Bale, 1989). La construcción desordenada de edificios no integrados al paisaje natural termina por suscitar el rechazo de quienes deberían utilizarlos. A su vez, los estadios producen un nuevo ecosistema en su entorno, crean polución acústica y hasta trastornos de orden público en sus alrededores; con el paso del tiempo, los precios de las viviendas y de los alquileres disminuyen en sus áreas de influencia (Bale, 1989).

La tendencias examinadas en la evolución del deporte durante el último decenio muestran como la diversificación del mismo es la característica prin- 
cipal. Asimismo, el deporte - junto con las actividades de ocio, en general-ha adquirido centralidad en la dinámica social contemporánea; es instrumento privilegiado en la autorrealización de las personas en este final de siglo. Las grandes tendencias para el año 2000 están definidas. Sin embargo, es más diff́cil precisar si éstas cambiarán sus orientaciones durante el próximo siglo. A nuestro entender, para conocer este aspecto debería profundizarse en dos caminos.

El primero, tal como se ha dicho, consiste en estudiar detalladamente los impactos que produce la transformación del sistema deportivo, así como la conexión que se da entre los mismos. De este modo será posible conocer efectos a largo plazo y posibles reacciones de la demanda. El segundo, consiste en interrogarse sobre la evolución de la centralidad del deporte a que nos hemos referido. ¿Seguirá siendo una de las actividades de ocio más valoradas por la población o, por el contrario, habrá otras más valoradas socialmente? En tal caso, si el interés se desplaza hacia otras ofertas culturales, el ritmo creciente que ha venido sufriendo el deporte en los últimos tiempos se detendrá; ¿dejará de ser "el mayor fenómeno de masas" que fue en la segunda mitad del siglo xx? Ante las dudas, ante los interrogantes, no queda otra vía que la de reorientar la ciencia del deporte convirtiéndola en una ciencia "abierta, significativa y planificada" capaz de dar respuesta a los retos que plantea el conocimiento de nuestro futuro (Heinemann 1986, 1991).

\section{BIBLIOGRAFIA}

Andres Orizo, F. (1979), Cambio sociocultural y comportamiento económico, Madrid, Centro de Investigaciones Sociológicas.

Bate, J. (1989), Sports geography, London-New York, E. \& F.N. Spon.

Bell, D. (1976), El advenimiento de la sociedad industrial, Madrid, Alianza Editorial. Brohm, J.M. (1976), Sociologia politica del deporte, México, Fondo de Cultura Económico.

Burriel, J.C. (1991), "Perspectivas en el diseño de las políticas deportivas munici" pales" en Actas del congreso Politicas deportivas e investigacion social, Pamplona, Gobierno de Navarra, Departamento de Educación Cultura y Deporte.

Cagigal, J.M. (1983), "El cuerpo y el deporte en la sociedad moderna" en Papers. Revista de Sociologia, n. 22, pág. 145-156.

Callede, J.P. (1984), Dynamique spatiale et politiques d'équipements. Le cas des équipements culturels et sportiff: leur rôle dans les rapports centre-péripherie, Grenoble, Association française de sciences politiques, Ile. Congrés National.

Consejo Superior de Deportes (1991), Encuesta sobre la estructura ocupacional del deporte en los sectores de entrenamiento, docencia, animación y dirección, Madrid. 
"Papers": Revista de Sociologia

Realizada por Jesús Martínez del Castillo, Núria Puig, Antonio Fraile y Agustín Boixeda.

Dietrich, K.; Heinemann, K. (1989), Der Nicht-sporliche Sport, Schondorf, Hofmann Verlag.

Duran, M.A. (1987), La práctica del ejercicio fisico del ama de casa española. Un estudio sociologico en Ministerio de Cultura. Instituto de la Mujer, Mujer y deporte, Madrid, pág. 91-101

García Ferrando, M. (1982), Deporte y sociedad, Madrid, Ministerio de Cultura.

Garcfa Ferrando, M. (1986), Hábitos deportivos de los españoles (sociologia del comportamiento deportivo), Madrid, Ministerio de Cultura, Consejo Superior de Deportes.

García Ferrando, M.; Pérez de Guzmán, T. (1990), Los estilos de vida de los valencianos, Valencia, Generalitat Valenciana.

García Ferrando, M. (199la), "El conocimiento de la realidad deportiva española. Problemas teóricos y metodológicos" en Actas del congreso Politicas deportivas $e$ investigación social, Pamplona, Gobierno de Navarra, Departamento de Educación Cultura y Deporte.

García Ferrando, M. (19916), Los españoles y el deporte (1980-1990). Un análisis sociolbgico, Madrid, Ministerio de Educación y Ciencia, Consejo Superior de Deportes, 1991.

Heinemann, K. (1986), "The future of sports. Challenge for the science of sport" en International Review for the Sociology of Sport, n. 4, vol. 21, pp. 271-285.

Heinemann, K.; Becker, H. ed. (1987), Die Zukunft des Sports, Schondorf, Hofmann Verlag.

Heinemann, K. (1991), "Tendencias de la investigación social aplicada al deporte" en Actas del congreso Politicas deportivas e investigación social, Pamplona, Gobierno de Navarra, Deparcamento de Educación Cultura y Deporte.

Heinemann, K. (1992), Die Wirtschoft des Sparts. Eine Einführung, Schondorf, Hofmann Verlag, en preparación.

Heinila, K. (1989), "The sports club as a social organization in Finland" en International Review for the Sociology of Sport, n. 3, vol. 24, pag. 225-248.

Horch, H-D. (1989), "Sociological research on sport organisations in the Federal Republic of Germany» en International Review for the Sociology of Sport, n. 3, vol. 24, pág. 201-216.

Institut d'Estudis Metropolitans (1986), Enquesta Metropolitana 1985-1986. ACtivitats $i$ formes de vida de la poblacib. Informe I. Resultats generals, Bellaterra. Realizada por María Jesús Izquierdo, Faustino Miguélez y Marina Subirats.

Jimenez, E. y col. (1990), "Associativisme juvenil a Barcelona" en Jove 2000, $(20 / 21)$.

Koski (1991), Ideology as an element in Sports Clubs, comunicación presentada en el "Segundo Congreso Mundial de Ciencias del Deporte", Barcelona.

Malenfant, Ch. (1987), "Sociabilities and volunteering in sports associations in Francen en International Review for the Sociology of Sport, n. 4, vol. 22, pág. 281-294. 
Malenfant, Ch. (1989), "Sociolgy of sports organisations in France" en International Review for the Sociology of Sport, n. 3, vol. 24, pág. 217-224.

Metoudi, M. (1987), "Les leçons de la publicite" en Le nouvel age du sport, monográfico de "Esprit".

Puig, N. (1990), "El futuro del deporte: el deporte en el año 2000" en Cuadernos Técnicos de Deporte, n. 4, pág. 85-95.

Puig, N.; Burriel, J.C. y col. (1990), L'esport com a generador de mobilitat i estructurador de l'espai en Zatagoza, A.; Puig, N., Oci, esport i societat, Barcelona, PPU, pág. 187-203.

Puig, N. (1992), Joves i esport: influència dels processos de socialització en els itineraris esportius de la població juvenil, Barcelona. Tesi doctoral en preparació.

Slack, T.; Kikulis, L.M. (1989), "The sociological study of sport organizations: some observations on the situation in Canadas en International Review for the Sociology of Sport, n.3, vol.24, pág. 179-200.

Vázquez, B. (1990), La presencia de la mujer en el deporte español, informe presentado en el seminario "Mujer y deporte", Madrid, Consejo Superior de Deportes, Instituto de la Mujer. 